\title{
Risk Factors Affecting the Phenotypic Expression of Heterozy- gous Familial Hypercholesterolemia in Pakistani Population
}

\author{
Fauzia Imtiaz', Wajahatullah Khan ${ }^{2 *}$, and Ahsan A. Vahidy ${ }^{3}$
}

${ }^{1}$ Department of Biochemistry, Dow International Medical College, Dow University of Health Sciences, Baba-EUrdu Road, PO BOX 942,943, Karachi 74200, Pakistan. ${ }^{2}$ Biochemistry Department, King Saud University, PO Box 2455, Riyadh 11451, Saudi Arabia. ${ }^{3}$ Department of Genetics, University of Karachi, Karachi Pakistan.

Received: February 16, 2010 / Accepted: May 27, 2010

\section{Abstract}

$\mathbf{F}$ amilial hypercholesterolemia (FH) is a genetic disorder of lipoprotein metabolism recognized by high plasma concentrations of low-density lipoprotein cholesterol (LDLC), tendon xanthomas, and high risk of early coronary heart disease. The phenotypic expression of $\mathrm{FH}$ is variable and several genetic and other risk factors contribute to such variability in clinical expression of the disease. FH is an autosomal disorder characterized by increased levels of total cholesterol (TC), low density lipoprotein cholesterol (LDL-C) and tendinous xanthmata (TX). Clinical phenotype of FH has previously been shown to be associated with increased coronary heart disease (CHD) and premature death. The present study was carried out in a Pakistani population to examine the contribution of environmental factors on the expression of this disease. A detailed examination of the physical and biochemical features of the FH was performed in a cohort of 335 individuals (202 males, 133 females) from Pakistan. Our results indicate that Pakistani females with FH had high levels of TC and LDL-C as compared to Pakistani males. Overall the concentrations of high density lipoprotein cholesterol (HDL-C) were significantly lower for both sexes as compared to the controls (normal). We have also found that high cholesterol levels were associated with increased incidence of CHD in FH Pakistani population where both male and female individuals showed $69 \%$ and $31 \%$ CHD, respectively. The mean onset age for coronary symptoms was about 38 years in males as compared to $\mathbf{4 5}$ years in females. A greater risk of developing CHD has been linked with levels of TC along with a history of smoking in males and presence of hypertension (HTN) in females. Our results indicate that these risk factors possibly affect and contribute in the phenotypic expression of FH in Pakistani population.

* Corresponding author: wkhan@ksu.edu.sa

Keywords: Phenotypic expression, Familial hypercholesterolemia, Risk factors, Coronary heart disease, Smoking, Hypertension, Total Cholesterol, LDL-C

\section{Introduction}

Familial hypercholesterolemia (FH) is a genetic disorder associated with lipoprotein metabolism, where elevated levels of low-density lipoprotein cholesterol (LDL-C) occur due to a mutation in the LDL receptor gene (Austin et al., 2004; Kwiterovich, 2008) to which LDL-C bind and remove it from the bloodstream. However, people with FH have less LDL receptors which result in increased than normal levels of LDL-C in the blood. Transmitted in an autosomal dominant fashion, $\mathrm{FH}$ is also characterized by the presence of tendinous xanthomas (TX) and premature atherosclerosis. (Alonso et al., 2009). FH is also one of the most common inherited diseases in the world, with a worldwide frequency of 1 in 500 for heterozygotes and 1 per million for homozygotes or compound heterozygotes (Goldstein et al., 2001). However, a higher incidence rate can be found in certain populations, for example the Afrikaners, Christian Lebanese, Finns, and French-Canadians, because of the founder effects (Goldstein et al., 2001). Patients with heterozygous FH express high levels of plasma LDL-C at a young age, causing atherosclerosis and an increased risk of cardiovascular diseases (Austin et al., 2004). Heterozygous FH patients show about two- to three-fold increase in LDL-C concentrations with a decrease in high-density lipoprotein (HDL-C) levels, along with tendinous xanthomatosis and premature heart disease (CHD) between the age of 35 and 55 years (Gagné et al., 1979; Civeira, 2004). About half of the offspring of an affected parent will have an increased level of cholesterol in the plasma and both genders are equally 
affected. Although, a large number of $\mathrm{FH}$ cases are due to the mutations in the LDL receptor gene (LDLR), additional contributory genes have also recently been reported for FH. Nevertheless, the mutations in these genes appear to be rare in the populations that have so far been investigated (Abifadel et al., 2003; Rader et al., 2003; Damgaard et al., 2004; Graham et al., 2005).

Advancing age and male gender are strongly associated with CHD risk, with men typically developing disease symptoms 10-15 years earlier than women who generally do not show such symptoms until after menopause (AHA, 2001). However, the course of $\mathrm{CHD}$ symptom in $\mathrm{FH}$ is different, with some patients expressing events earlier than others inspite of similar increased LDL-C levels. Hence other factors may play a part in CHD risk in these individuals. Earlier studies in European and North-American populations have established the influence of risk factors like age, smoking and hypertension (HTN) in FH individuals (Civeira, 2004). Among the environmental risk factors, smoking is the major contributor and has been associated with a nearly two fold higher risk (Hill et al., 1991). Lack of exercise and the related adiposity, including high intake of saturated fats and a reduced intake of certain vitamins have also been linked with increased risk (AHA, 2001). The mechanism of action of these factors is by determining the differences in the plasma levels of lipids and lipoproteins that are atherogenic and high levels of LDL-C and low levels of HDL-C have been associated with CHD (Castelli et al., 1986).

Evidence for strong genetic component for CHD risk is supported by the association between a family history of early $\mathrm{CHD}$ and a personal increased risk (Kardia et al., 2003) in the order of 1.7-fold higher (Hawe et al., 2003). The clinical phenotype for homozygotes is more severe than heterozygotes; however the expression of heterozygous $\mathrm{FH}$ is highly variable probably due to other risk factors in addition to the genes affecting the lipoprotein metabolism (Bertolini et al., 2004).

We have previously reported that the cause of $\mathrm{FH}$ in Pakistani population is partly due to familial resemblance contributing due to the genes but other risk factors may also play a role in the progression of the $\mathrm{FH}$ disease in Pakistani population (Imtiaz, 2009). In the present study, we studied and assessed the contribution of risk factors on the phenotypic expression of $\mathrm{FH}$ disease in a Pakistani population.

\section{Materials and Methods}

The study, upon written informed consent approval, included a group of 1523 unrelated patients with the clinical diagnosis of heterozygous $\mathrm{FH}$. A detailed examination of the physical and biochemical features of $\mathrm{FH}$ was obtained in this cohort.

\section{Clinical Examination \\ Physical FH Characteristics}

The physical characteristics that were examined in $\mathrm{FH}$ patients included presence of tendinous xanthoma (TX), xanthelesma, Arcus cornea (AC) and polyarthritis. A xanthelasma is a clearly distinguishable yellowish collection of cholesterol underneath the skin, typically on or in the region of the eyelids. The xanthelasma is a distinctive condition and is called a xanthoma when it becomes bigger and nodular. The TX is clinically characterized by papules and nodules found in the tendons of the hands. The AC is due to the white arc appearance on the cornea as a result of abnormal deposits of phospholipids and cholesterol. The polyarthritis is a condition when any type of arthritis involving five or more joints of the body was present in patients.

Three hundred thirty-five (335) subjects fitting to "definite FH" criteria had increased levels of LDL-C, presence of early heart attacks in the family and tendon xanthomatosis. Five probands were examined and their family pedigree trees were constructed to observe the dominant inheritance pattern of the disease.

\section{Biochemical Analysis}

The biochemical tests were performed following overnight (12-14 hrs) fasting. About $5 \mathrm{~mL}$ blood samples were drawn to analyze the total cholesterol (TC) levels, triglyceride (TG), HDL and LDL. The concentration of serum cholesterol, HDL-C, and TG were determined enzymatically by the CHOD-PAP and GPOPAP methods (Boehringer Mannheim, Germany) (Tietz, 1986). The HDL-C was isolated from serum by heparin manganese precipitation of the other lipoproteins. All the lipid profile analysis of TC, HDL-C, LDL-C and TG concentrations were observed in the serum of all 1523 Pakistani individuals diagnosed with $\mathrm{FH}$, cholesterol content of the serum LDL-C was estimated using Friedewald formula (Friedewald et al., 1972).

\section{Statistical Analysis}

The obtained data were analyzed for correlation among different risk factors using the Statistical Package for Social Sciences version 12 (SPSS, Inc). Statistically significant differences between groups or among groups for continuous variables were evaluated using Student's t test for unpaired data and ANOVA respectively. The pedigree was made by using the computer based software Cyrillic version 2.10 (Oxford, UK).

\section{Results}

Our previous study on the heritability of Pakistani FH population showed that the genetic factors are the major determinant of $\mathrm{FH}$, however because heritability is the proportion of variation due to additive familial effects of genetic and nongenetic sources, therefore we concluded that other non-genetic risk factors may also contribute in the progression of the disease (Imtiaz, 2009). The present study was conducted to examine the involvement of risk factors such as age, sex, HTN, smoking and TC concentrations in the phenotypic expression of heterozygous familial hypercholesterolemia in a cohort of Pakistani population living in the city Karachi. We have studied a total of 1523 unrelated patients with the clinical diagnosis of heterozygous FH. Out of these 1523, only 335 subjects with "definite $\mathrm{FH}$ " diagnosis belonged to five probands with cholesterol level $>300 \mathrm{mg} \mathrm{dl}^{-1}$ (normal range 140-240 mg dl${ }^{-1}$ ). Among them 
202 were males and 133 were females. Upon clinical physical examination, out of these 335 individuals (202 males and 133 females) $224(70 \%)$ showed TX. A total of 146 males $(72 \%)$ and 78 females (58\%) aged between $30-40$ showed TX symptoms (Table 1). Xanthelesma was detected in 258 individuals $(80 \%)$, i.e. 148 males (73\%; aged $40-50)$ and 110 females ( 82 $\%$; aged 30-40). The AC was observed in a total of 224 patients $(70 \%)$, where $63 \%$ males (128 individuals) aged $40-50$ and $72 \%$ females (96 individuals) aged $30-40$ showed the AC symptoms. The polyartheritis symptoms were less common among the observed individuals and only 7 individuals $(2 \%)$ have this condition. Among the 202 males (aged 40-50), only 2 males $(0.9 \%)$ and 5 females $(3.7 \%)$ showed the clinical symptoms of polyartheritis (Table 1). Hence, the occurrence of xanthoma (i.e. tendinous xanthomata and xanthelesma) in the present study in general was about $70 \%$ in the patients diagnosed with $\mathrm{FH}$.

A detailed examination of the physical and biochemical features of FH of cohort of 208 females and 156 males showed that females with FH had higher levels of total LDL-C (196 to $358 \mathrm{mg} \mathrm{dl}^{-1}$ ) compared to the male subjects (201 to $301 \mathrm{mg} \mathrm{dl-}$
1) (Figure 1), while having lower HDL-C levels (25-55 $\mathrm{mg} \mathrm{dl}^{-1}$ ) in both genders compared to the normal (Figure 1).

Our results also showed that the mean age of $\mathrm{FH}$ showing the symptoms in males was earlier than normal, i.e. 38 years compared to 45 years in females. The $\mathrm{FH}$ disease prevalence rate was $69 \%$ in males compared to $31 \%$ in females. The risk factors such as total cholesterol (TC), smoking and HTN were found to be highly correlated with $\mathrm{FH}$ appearance. We observed that smoking and HTN were significantly $(p<0.05)$ correlated. Similarly, a high correlation ( $p<0.05$ ) was found between smoking and TC in the individuals with FH. However, TC and HTN were even more strongly correlated with FH at $\mathrm{p}<0.01$ (Table 2). The risk factors such as smoking and HTN exhibited significant effects in the appearance of the disease. Our study showed that smoking and HTN were significantly correlated with the level of total cholesterol at $p<0.05$ (0.047) and $p<0.01$ (0.000), respectively (Table 2). The smoking in males had profound effect in observed individuals, where we noticed that $70 \%$ male smokers were positive for CHD.

Fig. 1. Pedigree showing the presence of FH in every generation of the proband confirming the dominant inheritance pattern. The amount of LDL-C is mentioned as $\mathrm{mg} / \mathrm{dL}$.

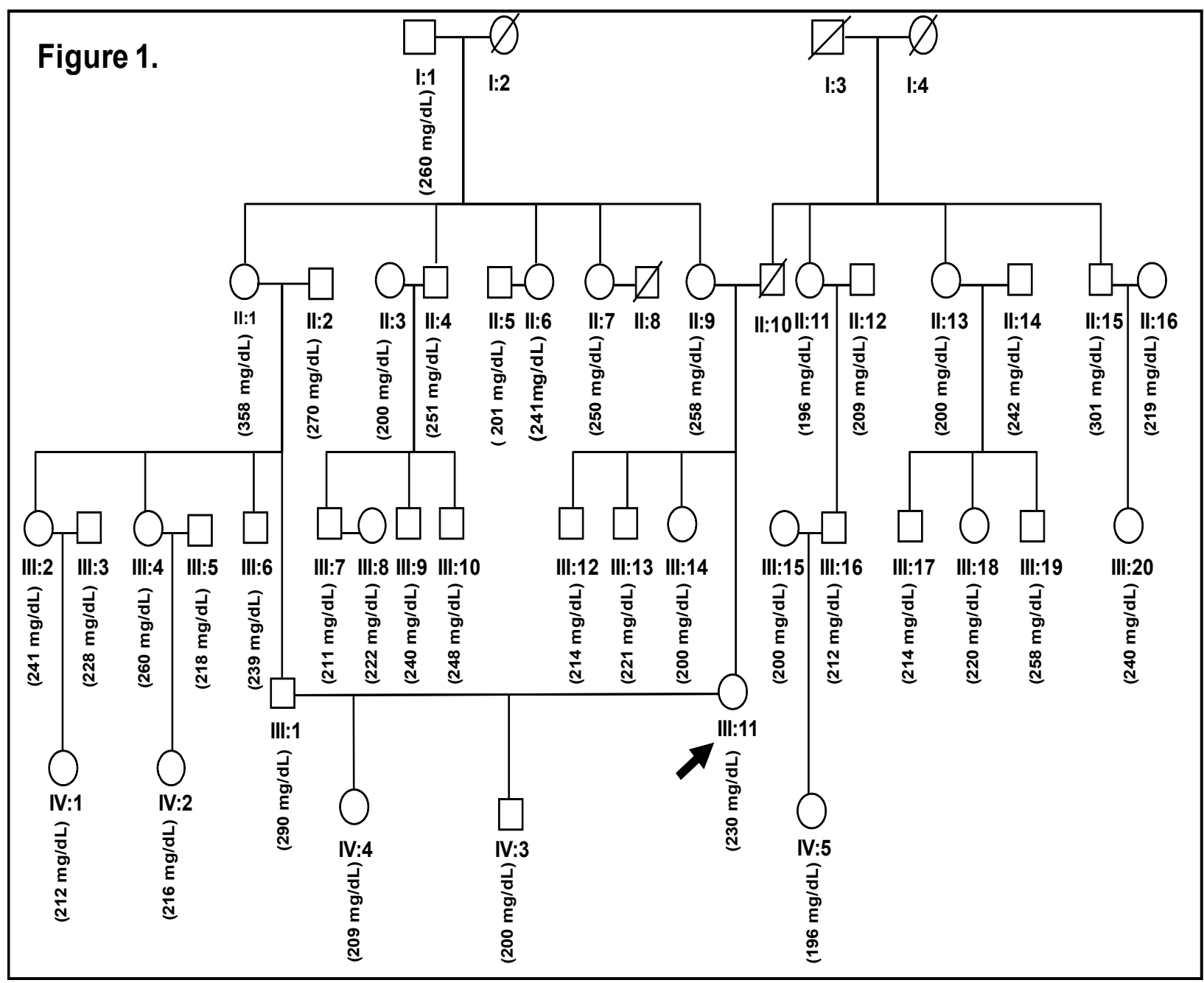


Table 1. Clinical features of 335 participants with cholesterol level $>210 \mathrm{mg} / \mathrm{dL}$ in Pakistani population.

\begin{tabular}{|c|c|c|c|c|c|}
\hline Observed Features & Gender & Age (Years) & Total & Observed Cases & Percentage (\%) \\
\hline \multirow{3}{*}{ Tandinous Xanthomata } & \multirow{3}{*}{ Male } & $30-40$ & 202 & 146 & 72 \\
\hline & & $40-50$ & 0 & 0 & 0 \\
\hline & & $50-60$ & 0 & 0 & 0 \\
\hline & \multirow{3}{*}{ Female } & $30-40$ & 133 & 78 & 58 \\
\hline & & $40-50$ & 0 & 0 & 0 \\
\hline & & $50-60$ & 0 & 0 & 0 \\
\hline \multirow{6}{*}{ Xanthelesma } & \multirow{3}{*}{ Male } & $30-40$ & 0 & 0 & 0 \\
\hline & & $40-50$ & 202 & 148 & 73 \\
\hline & & $50-60$ & 0 & 0 & 0 \\
\hline & \multirow{3}{*}{ Female } & $30-40$ & 133 & 110 & 82 \\
\hline & & $40-50$ & 0 & 0 & 0 \\
\hline & & $50-60$ & 0 & 0 & 0 \\
\hline \multirow{6}{*}{ Arcus cornea } & \multirow{3}{*}{ Male } & $30-40$ & 0 & 0 & 0 \\
\hline & & $40-50$ & 202 & 128 & 63 \\
\hline & & $50-60$ & 0 & 0 & 0 \\
\hline & \multirow{3}{*}{ Female } & $30-40$ & 133 & 96 & 72 \\
\hline & & $40-50$ & 0 & 0 & 0 \\
\hline & & $50-60$ & 0 & 0 & 0 \\
\hline \multirow{6}{*}{ Polyarthritis } & \multirow{3}{*}{ Male } & $30-40$ & 0 & 0 & 0 \\
\hline & & $40-50$ & 202 & 2 & 0.9 \\
\hline & & $50-60$ & 0 & 0 & 0 \\
\hline & \multirow{3}{*}{ Female } & $30-40$ & 0 & 0 & 0 \\
\hline & & $40-50$ & 0 & 0 & 0 \\
\hline & & $50-60$ & 133 & 5 & 3.7 \\
\hline
\end{tabular}

Table 2. Correlation studies showing relations among smoking, hypertension (HTN) and Total cholesterol levels in Pakistani population.

\begin{tabular}{|l|c|c|c|}
\hline & Smoking & HTN & Total Cholesterol \\
\hline Smoking & 1.000 & $0.32^{*}$ & $0.47^{*}$ \\
\hline HTN & & 1.000 & $0.000^{* *}$ \\
\hline Total Chlesterol & & & 1.000 \\
\hline
\end{tabular}

Significant at $* p<0.05, * * p<0.01$.

\section{Discussion}

$\mathrm{FH}$ is a public health problem throughout the world and many heterozygous $\mathrm{FH}$ remain undetected until adulthood. The present study was conducted to study the contribution of risk factors (age, sex, smoking and HDL-C concentrations) in the phenotypic expression of heterozygous $\mathrm{FH}$ in a Pakistani population. $\mathrm{FH}$ may also cause premature coronary artery disease and atherosclerotic plaque formation and is considered as one of the first inherited disorders as being a cause of myocardial infarction (heart attack). In the present study, out of a total of 1523 unrelated patients with the clinical diagnosis of heterozygous $\mathrm{FH}$, only 335 individuals diagnosed with $\mathrm{FH}$ belonged to five probands (202 males and 133 females). Similar criteria have previously been used to identify patients with $\mathrm{FH}$ based on high plasma levels of total and LDL cholesterol, family history of hy- percholesterolemia, tendon xanthomas or corneal arcus, and personal and family history of premature CHD (Goldstein et al., 2001). The FH patients have LDL-C levels almost twice than the normal population ( 190 to $400 \mathrm{mg} \mathrm{dl}^{-1}$ ).

The clinical examination of the 335 individuals with $\mathrm{FH}$ showed that $72 \%$ males and $58 \%$ females had TX at ages between $30-40$ years. Xanthelesma was found in $80 \%$ males (aged 40-50) and in $82 \%$ females (aged 30-40). The AC was observed in 63\% males (aged $40-50$ ) and $72 \%$ females (aged $30-40$ ). A very few individuals ( $2 \%$ i.e. 7 individuals) showed polyartheritis symptoms among them were only 2 males $(0.9 \%)$ aged 40-50; while 5 females (3.7\%) showed the disease symptoms. The TX is pathognomonic of $\mathrm{FH}$ but its identification is not always straightforward and to some extent considered insensitive diagnostic markers. However, other criteria should be taken into consideration for the diagnosis of $\mathrm{FH}$ including personal and familial LDL-C levels, history of CHD, and presence of CA before the age of 45 years and xanthomas (Fernando, 2004). Overall, the incidence of xanthoma (i.e. tendinous xanthomata and $x$ anthelesma) in the present study was quite high i.e. 7 out of $10(70 \%)$ cases with FH had it. Recently, Junyent et al. (2005) has reported that the occurrence of TX is very much linked to the $\mathrm{FH}$ incidences in individuals with family history of hypercholesterolemia, primary hypercholesterolemia and premature coronary disease. Although TX is pathognomonic of $\mathrm{FH}$ but a high variability of xanthoma may occur in $\mathrm{FH}$ patients (Descamps et al., 2001). Xanthelasmas occur commonly in heterozygotes, and are rare in homozygotes. The presence of xanthelasmas is not specific for $\mathrm{FH}$ and may also occur in individuals with normal lipid levels (Goldstein et al., 2001). The frequency of FH among Caucasians is 1:500, however in some populations there is high heterozygous FH frequency such as French Canadians, Lebanese Christians, South African Afrikaners, Lithuanian Ashkenzai Jews, Druze and Finns (Lehrman et al., 1987; Kotze et al., 1991; Meiner et al., 1991; Leitersdorf et al.,1990; Landsberger et al., 1992; Koivisto et al., 1992). The heterozygote FH individuals have about two fold increase in their plasma cholesterol. The $\mathrm{FH}$ subjects show 4-5 time higher age-sex standardized mortality ratios in the general populations (Castro-Orós et al., 2010).

The mean age of males that showed the symptoms of $\mathrm{FH}$ in the present study was 38 years, compared to 45 years in females. The incidence rate in the observed males was $69 \%$ compared to $31 \%$ in females. Similar results have previously been reported where the observed age was strongly associated with high cholesterol levels in men, whereas women had significantly lower non-HDL-C level (Gardner et al., 2000). For women, the age range was 25 to 64 years, whereas for men it ranged from 25 to 54 years. Non-HDL concentration was higher in individuals with increasing age. However, this observation seemed to be not influenced by age as the total cholesterol levels were found to be higher in all age groups. The mean age of males, showing symptoms of $\mathrm{FH}$ was $38 \pm 5$ years, which is earlier as compared to females ( $45 \pm 5$ years). The $\mathrm{FH}$ should immediately be treated upon diagnosis and if left untreated approximately $85 \%$ of males and $50 \%$ of females will develop a coronary problem before the age of 65 (Civeira, 2004). It is striking that up to $9 \%$ of the total premature CHD in eastern Germany and 
Finland has been linked with FH (Koivisto et al., 1993; Baron et al., 1996; Schuster, 2002). Hence, the major cause of death in $\mathrm{FH}$ patients has been linked with the occurrence of CHD (Mabuchi et al., 1989; Miettinen and Gylling, 1988). In the present study, we found that the high levels of LDL-C (> $190 \mathrm{mg} \mathrm{dl}^{-1}$ ) were detected in the proband and in the related family members (Figure 1). The clinical diagnosis of heterozygote $\mathrm{FH}$ is associated not only with the high plasma level of LDL-C but also with the incidence of other coronary risk factors (Hill et al, 1991). We have found that the females with $\mathrm{FH}$ had higher levels of total LDL-C (196-358 $\left.\mathrm{mg} \mathrm{dl}^{-1}\right)$ as compared to the male subjects (201-301 $\left.\mathrm{mg} \mathrm{dl}^{-1}\right)$ and with lower levels of HDL-C (25-55 $\left.\mathrm{md} \mathrm{dl}^{-1}\right)$. The average age of onset of coronary symptoms was delayed in females, with a mean age of 55 years compared with 48 years for males $(p<0.05)$. Previous studies have shown an association between clinical heterozygous $\mathrm{FH}$ and coronary heart disease (Kalina et al., 2001; Umans-Eckenhausen et al., 2002). However, the heterogeneity in $\mathrm{FH}$ patients with regards to plasma LDL-C and CHD has also been suggested (CastroOrós et al., 2010). Multivariate analyses have revealed that in $\mathrm{FH}$ heterozygotes and controls, the HDL-C levels may contribute to a greater proportion of the variation in TC to HDL-C ratio than TC (Torres et al., 1996).

A number of factors, including age, sex, smoking and HDL-C concentrations have previously been identified as risk factors for CHD in FH in cross-section studies worldwide (Jansen et al., 2004). There are 100 million people with $\mathrm{FH}$ worldwide, mainly heterozygotes, and approximately $85 \%$ of males and $50 \%$ of females with $\mathrm{FH}$ will suffer a coronary event before they are 65 years old, if appropriate preventive measures are not implemented (Fernando, 2004). The risk factors such as smoking and HTN showed a significant correlation in the appearance of the disease. Our study showed that a strong correlation between the risk factors, smoking and HTN, was present in the individuals with $\mathrm{FH}$. The TC and smoking were significantly correlated $(\mathrm{P}<0.05)$ in the FH Pakistani population. Similarly, smoking and HTN were also significantly $(P<0.05)$ correlated. But a very strong correlation ( $p<0.001$ ) between HTN and TC was observed in the studied individuals. Smoking in males had profound effect, where $70 \%$ males with positive CHD were smokers. However, the HTN in females was associated with higher incidence of CHD. The HTN has been considered as an independent risk factor for females with $\mathrm{FH}$ (Hill et al., 1991). It has been reported that the level of total cholesterol was positively related to the prevalence of systolic HTN and obesity, in addition to smoking (Rywik et al., 1999). A meta-analysis was conducted by Rywik et al. (1999), where they concluded that for every $10 \%$ reduction in TC, there was $2.5 \%$ decrease in the incidence of CHD. A multivariate analysis performed on a Canadian population indicated that the low HDL-C and smoking were the best predictors of risk and the average age of onset of coronary symptom were delayed in females (55 years) compared to males (48 years) (Weber et al., 1997). The risk of developing heart related problems has also been connected with the lower levels of HDL-C and a history of smoking; however, in women, the heart related problems were mainly associated with elevated TG levels and the presence of HTN (Hill et al., 1991; Neil et al., 2004). Our results are in agreement with earlier published reports (Vuario et al., 1997; Jansen, 2004) on the risk factors like age, sex, smoking and HTN. Our results are consistent with accumulating evidence from a number of cross-sectional population and case control studies that have assessed the role of established and emerging risk factors in individuals with $\mathrm{FH}$. This study on a Pakistani population has shown that $\mathrm{FH}$ is linked to risk factors such as age, smoking and HTN in addition to total cholesterol levels and to a larger extent was associated with the development of premature heart diseases as well as in the phenotypic expression in heterozygous $\mathrm{FH}$.

Upon diagnosis the $\mathrm{FH}$ patients should immediately be treated and if left untreated, they have a high chance (about 8 times) of early coronary heart disease (CHD). Cholesterol can be accumulated in the coronary arteries of the heart causing angina or heart attacks. Other factors that increase the risk of $\mathrm{CHD}$ are smoking and high blood pressure. However, $\mathrm{FH}$ is a very treatable condition which can be controlled with the use of cholesterol-lowering drugs combined with a healthy lifestyle. It is now known that people with $\mathrm{FH}$ who are treated can expect to have a life expectancy which is the same as the general population. Therefore it is vitally important to diagnose this condition as early as possible so that the right treatment can be started and heart attacks can be prevented.

\section{References}

Abifadel $M$, Varret $M$, Rabes JP, Allard $D$, Ouguerram K, Devillers $M$, Cruaud $C$, Benjannet $S$, Wickham L, Erlich D, Derre A, Villeger $L$, Farnier M, Beucler I, Bruckert E, Chambaz J, Chanu B, Lecerf JM, Luc G, Moulin P, Weissenbach J, Prat A, Krempf M, Junien C, Seidah NG, Boileau C (2003) Mutations in PCSK9 cause autosomal dominant hypercholesterolemia. Nat Genet. 34: 154-156.

Alonso R, Defesche JC, Tejedor D, Castillo S, Stef M, Mata N, GomezEnterria P, Martinez-Faedo C, Forga L, Pedro Mata (2009) Genetic diagnosis of familial hypercholesterolemia using a DNA-array based platform. Clin Biochem. 42 (9): 899-903.

Austin MA, CM Hutter, RL Zimmern, and SE Humphries (2004) Familial hypercholesterolemia and coronary heart disease: a HuGE association review: Am J Epidemiol 160: 421-429.

Baron H, Fung S, Aydin A, Bahring S, Luft FC, Schuster H (1996) Oligonucleotide ligation assay (OLA) for the diagnosis of familial hypercholesterolemia. Nat Biotechnol. 14:1 279-1282.

Bertolini S, L Pisciotta, L Di Scala, S Langheim, A Bellocchio, P Masturzo, A Cantafora, S Martini, M Averna, G Pes, C Stefanutti, and S Calandra (2004) Genetic polymorphisms affecting the phenotypic expression of familial hypercholesterolemia. Atherosclerosis 174 (1): 57-65.

Castelli WP, RJ Garrison, PWF Wilson, RD Abbott, S. Kalousdian, and W. B. Kannel (1986) Incidence of coronary heart disease and lipoprotein cholesterol levels. The Framingham Study. JAMA 256: 2835 2838.

Castro-Orós ID, Pocoví M, Civeira F (2010) The genetic basis of familial hypercholesterolemia: inheritance, linkage, and mutations. The Application of Clinical Genetics 3: 53-64

Civeira F (2004) International Panel on Management of Familial Hypercholesterolemia. Guidelines for the diagnosis and management of heterozygous familial hypercholesterolemia. Atherosclerosis. Mar; 173(1):55-68.

Damgaard D, Jensen JM, Larsen ML, Soerensen VR, Jensen HK, Gre- 
gersen N, Jensen LG, Faergeman $O$ (2004) No genetic linkage or molecular evidence for involvement of the PCSK9, ARH or CYP7A1 genes in the familial hypercholesterolemia phenotype in a sample of Danish families without pathogenic mutations in the LDL receptor and apoB genes. Atherosclerosis. 177: 415-422.

Descamps OS, Leysen X, Van Leuven F, Heller FR. The use of Achilles tendon ultrasonography for the diagnosis of familial hypercholesterolemia. Atherosclerosis. $2001 ; 157: 514-518$.

Doll $R$, and $A B$ Hill (1966) Mortality of British doctors in relation to smoking: observations on coronary thrombosis. J Natl Cancer Inst Monogr 19: 205-268.

Fernando C (2004) Guidelines for the diagnosis and management of heterozygous familial hypercholesterolemia. Atherosclerosis 173: 55-68.

Friedewald WT, RI Levy, and DS Fredickson (1972) Estimation of the concentration of low density lipoprotein cholesterol in plasma without use of preparative ultracentrifuge. Clin Chem 18: 499-502.

Gagn'e C, Moorjani S, Brun D, Toussaint M, Lupien PJ. (1979) Heterozygous familial hypercholesterolemia. Relationship between plasma lipids, lipoproteins, clinical manifestations and ischaemic heart disease in men and women. Atherosclerosis 34:13-24.

Gardner CD, Marilyn AW, Stephen PF (2000) Population frequency distribution of non-high-density lipoprotein cholesterol (third national health and nutrition examination survey [NHANES iii], 1988-1994). Am J Cardiol 86: 299-304.

Goldstein JL, Brown MS (1974) Binding and degradation of low density lipoproteins by cultured human fibroblasts. Comparison of cells from a normal subject and from a patient with homozygous familial hypercholesterolemia. J Biol Chem 249: 5153-5162.

Goldstein JL, Hobbs HH, Brown MS (2001) Familial hypercholesterolemia. In: Scriver CR, AL Beaudet, WS Sly, and D Vale (eds.) The metabolic and molecular bases of inherited disease, Vol. 120, New York, NY: McGraw-Hill Companies, Inc, pp. 2863-2914.

Graham CA, Mcllhatton BP, Kirk CW, Beattie ED, Lyttle K, Hart P, Neely RD, Young IS, Nicholls DP (2005) Genetic screening protocol for familial hypercholesterolemia which includes splicing defects gives an improved mutation detection rate. Atherosclerosis 182: 331-340.

Hawe E, Talmud PJ, Miller GJ, Humphries SE (2003) Family history is a coronary heart disease risk factor in the Second Northwick Park Heart Study. Ann Hum Genet 67: 97-106.

Heart and stroke facts. Dallas, TX (2001) American Heart Association. Hill JS, Hayden MR, Frohlich J, Pritchard PH (1991) Genetic and environmental factors affecting the incidence of coronary artery disease in heterozygous familial hypercholesterolemia. Arterioscler Thromb Vasc Biol 11 (2): 290-297.

Imtiaz F (2009) Estimation of heritability of familial hypercholesterolemia among 335 family members of five hypercholestrolemic probands of Pakstani population. J. Ayub Med Coll Abbottabad 21: 58-61.

Jansen ACM, Van Aalst-Cohen ES, Tanck MW, Trip MD, Lansberg PJ, Liem AH, Roeters van Lennep HWO, Sijbrands EJG, Kastelein JJP (2004) The contribution of classical risk factors to cardiovascular disease in familial hypercholesterolemia: data in 2400 patients. J Intern Med 256: 482-490.

Junyent M, Gilabert R, Zambón D (2005) The use of Achilles tendon sonography to distinguish familial hypercholesterolemia from other genetic dyslipidemias. Arterioscler Thromb Vasc Biol. 25: $2203-$ 2208.

Kalina A, Csaszar A, Czeizel AE, Romics L, Szaboki F, Szalai C, Reiber I, Nemeth A, Stephenson S, Williams RR (2001) Frequency of the R3500Q mutation of the apolipoprotein B-100 gene in a sample screened clinically for familial hypercholesterolemia in Hungary. Atherosclerosis 154: 247-251.
Kardia SL, SM Modell, PA Peyser (2003) Family-centered approaches to understanding and preventing coronary heart disease. Am J Prev Med 24: 143-151.

Koivisto UM, Hamalainen L, Taskinen MR, Kettunen K, Kontula K (1993) Prevalence of familial hypercholesterolemia among young north Karelian patients with coronary heart disease: a study based on diagnosis by polymerase chain reaction. J Lipid Res. 34:269-277.

Koivisto UM, Turtola H, Aalto-Setala K, Top B, Frants R R, Kovanen P T, Syvänen A C, Kontula K (1992) The familial hypercholesterolemia (FH)-North Karelia mutation of the low density lipoprotein receptor gene deletes seven nucleotides of exon 6 and is a common cause of FH in Finland. J Clin Invest. 90:219-228.

Kotze MJ, Langenhoven E, Warnich L, du Plessis L, Retief AE (1991) The molecular basis and diagnosis of familial hypercholesterolaemia in South African Afrikaners. Ann Hum Genet. 55:1 15-1 21.

Kwiterovich PO (2008) Primary and secondary disorders of lipid metabolism in pediatrics. Pediatr Endocrinol Rev;5(Suppl 2):727-38.

Landsberger D, Meiner V, Reshef A, Levy $Y$, van der Westhuyzen DR, Coetzee GA, Leitersdorf E (1992) A nonsense mutation in the LDL receptor gene leads to familial hypercholesterolemia in the Druze sect. Am J Hum Genet. 50:427-433.

Lehrman MA, Russell DW, Goldstein JL, Brown MS (1987) Alu-Alu recombination deletes splice acceptor sites and produces secreted low density lipoprotein receptor in a subject with familial hypercholesterolemia. J Biol Chem. 262:3354-3361.

Leitersdorf E, Tobin EJ, Davignon J, Hobbs HH (1990) Common lowdensity lipoprotein receptor mutations in the French Canadian population. J Clin Invest. 85:1014-1023.

Mabuchi H, Koizumi J, Shimizu M, Takeda R (1989) Development of coronary heart disease in familial hypercholesterolemia. Circulation. 79:225-232.

Meiner V, Landsberger D, Berkman N, Reshef A, Segal P, Seftel HC, van der Westhuyzen DR, Jeenah MS, Coetzee GA, Leitersdorf E (1991) A common Lithuanian mutation causing familial hypercholesterolemia in Ashkenazi Jews. Am J Hum Genet. 49:443-449.

Miettinen TA, Gylling H (1988) Mortality and cholesterol metabolism in familial hypercholesterolemia. Long-term follow-up of 96 patients. Arteriosclerosis. 8:163-167.

Müller C (1938) Xanthomata, hypercholesterolemia, angina pectoris: Acta Med Scand 89: 75-84.

Neil HAW, V Seagroatt, DJ Betteridge, M P Cooper, P N Durrington, J P Miller, M Seed, R P Naoumova, G R Thompson, R Huxley, and S E Humphries (2004) Established and emerging coronary risk factors in patients with heterozygous familial hypercholesterolemia. Heart 90: 1431 - 1437 .

Rader DJ, Cohen J, Hobbs HH (2003) Monogenic hypercholesterolemia: new insights in pathogenesis and treatment. J Clin Invest. 111 : 1795-1803.

Rywik SL, AM Teri, P Andrzej, P Walerian, ED Clarence, BB Grazyna and K Ewa (1999) Association of lipids and lipoprotein level with total mortality and morbidity caused by cardiovascular and cancer disease (Poland and United States Collaborative Study on Cardiovascular Epidemiology). Am J Cardiol 84: 540-548.

Schuster H (2002) High risk/high priority: familial hypercholesterolemia -a paradigm for molecular medicine. Atheroscler Suppl. 2:27-30.

Tietz NW (1986) Textbook of Clinical Chemistry, Philadelphia, PA, WB, Saunders Co. pp. 888.

Torres AL, S Mooriani, MC Vohl, C Gagne, B Lamarche, LD Brun, PJ Lupien, and JP Despres (1996) Heterozygous familial hypercholesterolemia in children: low-density lipoprotein receptor mutational analysis and variation in the expression of plasma lipoprotein-lipid concentrations. Atherosclerosis 126 (1): 163-71.

Umans-Eckenhausen MA, EJ Sijbrands, JJ Kastelein, and JC Defesche 
(2002) Low-density lipoprotein receptor gene mutations and cardiovascular risk in a large genetic cascade screening population. Circulation 106: 3031-3036.

Vuario AF, H Turtola, and KM Piilahti (1997) Familial hypercholesterolemia in the Finnish north Karelia. A molecular, clinical and genealogical study. Arterioscler Thromb Vasc Biol 17: 31 27-3138.
Weber M, S McNicoll, M Marcil, P Connelly, S Lussier-Cacan, J Davignon, Y Latour, and J Genest Jr (1997) Metabolic factors clustering, lipoprotein cholesterol, apolipoprotein (a) and apolipoprotein E phenotypes in premature coronary artery disease in French Canadians. Can J Cardiol 13 (3): 253- 260. 